\title{
The transition process of aerosol distribution state with spatio- temporal analysis based on MODIS product
}

\author{
Zhang Hong ${ }^{a b c}$ Su Weiqing ${ }^{a b c}$ Chen Li Long ${ }^{a b c}$ \\ ${ }^{a}$ Key Laboratory of Virtual Geographic Environment, Ministry of Education of PRC, Nanjing Normal \\ University, Nanjing 210023, China \\ ${ }^{b}$ Jiangsu Centre for Collaborative Innovation in Geographical Information Resource Development and \\ Application, Nanjing 210023, China \\ ${ }^{c}$ School of Geography Science, Nanjing Normal University, Nanjing 210023, China
}

Keywords: Aerosol, State transition, nonlinear analysis

\begin{abstract}
:
Atmospheric aerosol can have an impact on the climate through direct and indirect radiative forcing and cause air quality problems to harm human health [Charlson, R.J., et al. 1992]. Many scholars have made a lot of contributions to aerosol concentration analysis, but the analysis of chaotic aerosol concentration time series is always unable to perfectly solve the inherent randomness brought by complicated dynamic behaviors [Abarbanel, H.D.I, 1996]. This research has established the aggregation and dispersion states of aerosol optical thickness in fixed region and fixed time period by calculating spatial statistics of value-containing pixels in remote sensing images. And it has also analyzed the process of this state which is influenced by several meteorological factors. This kind of aggregation and dispersion is a conceptual method of spatial statistics in geography. The research compares the aerosol AOD value and its distribution area in the atmosphere from the signal frequency and utilizes the methods of nonlinear analysis and wavelet analysis to solve for its state transition characteristics at a regular point in time. Then the aerosol state transfer process is unfolded in space to analyze the interaction between the sequential meteorological factors and the aerosol state transfer process, as well as the comparison of the differences and similarities between the aerosol state transfer process in urban areas and non-urban areas. The research can not only provide theoretical foundation for pollution warning on the basis of long time series but also provide a new idea for analyzing the nonlinear dynamic process of the atmospheric system, which is the analysis of the change process of the chaotic system by spatial deploy at a specific time point.

The data used in this study include MODIS /terra and aqua MAIAC land aerosol optical Depth Daily products with a 1 $\mathrm{km}$ spatial resolution from January 2015 to December 2017 from NASA (accessed at http://ladsweb.nas.com,nasa. gov/ data/search.html). Accuracy Check Data come from ground-based aerosol observation data at six sites in eastern China of the global ground-based aerosol observation network from match 2015 to April 2017 (http://aeronet.gsfc.nasa.gov/cg i-bin/webtool_opera_v2_new). The meteorological data come from temperature, wind speed and direction, precipitation and humidity data of the National Meteorological Network of China (http:/ http://data.cma.cn/). The research region cov ers the largest urban agglomeration in China - the Yangtze river delta urban agglomeration $\left(28^{\circ} \mathrm{N} \sim 35^{\circ} \mathrm{N}, 117^{\circ} \mathrm{E} \sim 123^{\circ} \mathrm{E}\right)$. With a land area of 117,700 square kilometers, and the population being about 200 million, the economic zones are co mposed of 16 large- and medium-sized cities in Jiangsu Province and Zhejiang Province, which is the sixth largest urba $\mathrm{n}$ agglomeration in the world.

Through the pixel calculation of remote sensing image, the aerosol distribution area time series and aerosol AOD value time series were constructed. The intrinsic mode function and their remnants were obtained by EEMD decomposition of the two groups of normalized time series according to the mean value of the day. The sums of the intrinsic mode function and the trend term is the original function. Each intrinsic mode function represents the change process of aerosol concentration and aerosol distribution area at different time scales according to the frequency from high to low respectively. The residual quantifier is a monotone increasing signal, which represents the trend of aerosol AOD value and distribution area changing with time from 2015 to 2017. Then the Hilbert-Huang transformation was applied to two kinds of time series and the instantaneous frequency of two sets of time series on different time scales could be obtained.
\end{abstract}

Table 1 Intrinsic mode function of AOD value series and aerosol area series

\begin{tabular}{|c|c|c|c|c|c|}
\hline $\begin{array}{l}\text { Intrinsic mode } \\
\text { function }\end{array}$ & $\begin{array}{l}\text { AOD value IMF } \\
\text { variance }\end{array}$ & $\begin{array}{l}\text { AOD value IMF } \\
\text { contribution rate }\end{array}$ & $\begin{array}{c}\text { Aerosol area IMF } \\
\text { variance }\end{array}$ & $\begin{array}{l}\text { Aerosol area IMF } \\
\text { contribution rate }\end{array}$ & $\begin{array}{l}\text { Interdependency of } \\
\text { two time series }\end{array}$ \\
\hline Imf1 & $3.74 * 10^{-2}$ & $53.43 \%$ & $7.89 * 10^{-2}$ & $56.74 \%$ & 0.796 \\
\hline $\operatorname{Imf} 2$ & $1.18 * 10^{-2}$ & $16.97 \%$ & $2.14 * 10^{-2}$ & $15.39 \%$ & 0.701 \\
\hline $\operatorname{Imf} 3$ & $0.67 * 10^{-2}$ & $9.64 \%$ & $1.41 * 10^{-2}$ & $10.20 \%$ & 0.740 \\
\hline $\operatorname{Imf} 4$ & $0.46^{*} 10^{-2}$ & $6.65 \%$ & $0.97 * 10^{-2}$ & $6.98 \%$ & 0.794 \\
\hline Imf5 & $0.28 * 10^{-2}$ & $4.06 \%$ & $0.63 * 10^{-2}$ & $4.56 \%$ & 0.790 \\
\hline Imf6 & $0.26 * 10^{-2}$ & $3.76 \%$ & $0.53 * 10^{-2}$ & $3.83 \%$ & 0.819 \\
\hline Imf7 & $0.14 * 10^{-2}$ & $2.04 \%$ & $0.14 * 10^{-2}$ & $1.05 \%$ & 0.866 \\
\hline Imf8 & $0.22 * 10^{-2}$ & $3.24 \%$ & $0.15 * 10^{-2}$ & $1.13 \%$ & 0.433 \\
\hline Imf9 & $0.01 * 10^{-2}$ & $0.15 \%$ & $0.009 * 10^{-2}$ & $0.07 \%$ & 0.993 \\
\hline
\end{tabular}


According to the daily mean, there are ten intrinsic mode functions. Overall, AOD value and aerosol area have a good correlation, and the correlation becomes more significant with the increase of time scale. Through comparison, it can be found that the aerosol AOD value and the aerosol area distribution have consistency on a large time scale, but based on the day or shorter time unit, the area distribution is affected by the atmospheric system to produce different aggregation and dispersion states. And at a fixed point in time, the fixed region is principally dominated by a type of aggregation and dispersion state.

Setting days as the time scale, two constructed sets of time series are carried out on wavelet decomposition and singularity analysis. The singularity of general signal can be divided into two cases: 1 . At a certain moment, the amplitude of the signal mutates, causing incontinuity of the signal, and this type of mutation is called the mutant site of the first type; 2 . The signal appearance is very smooth and the amplitude is not mutated. However, the first-order differential of the signal is mutated and the first-order discontinuity occurs, this kind of mutation is called a second type of mutation point [konglinjun,et.al.2014] Statistics obtained 245 first-class mutation points and 134 second-class mutation points of aerosol concentration from 2015 to 2017. From 2015 to 2017, there were 271 first-class mutation points of the aerosol area distribution and 114 mutation points of the second class. The change of aerosol concentration dominated the change of area distribution through first-class mutation points, and multiple mutation of area distribution can occur in the concentration mutation interval. In the second-class mutation, the trend information of two sets of sequences is included. There are 87 state transition points of aerosol variation area distribution from 2015 to 2017 . On the basis of the singularity analysis of wavelet, the changes of two sets of sequences at different frequencies can be observed. When they have same first-class mutation points but different in second-class points, it shows that the change of aerosol area distribution at this time point is influenced by other factors and the state changes occur.

The spatial model of aerosol state transition was constructed based on the superposition of Moran index and meteorological vector field. Then, the Qualitative analysis of the state transition types and processes of aerosol spatial distribution was conducted, the states were divided into three categories: aggregation trend, dissipation trend and random distribution trend. Using the Quantitative method, the influence of four meteorological factors of temperature field, wind field, precipitation and humidity on each aerosol spatial state transition point were distinguished. From the perspective of process variation, the state transition process was still a nonlinear process, but the changing process of wind field and precipitation field totally dominated the state transition process of aerosol, which could transform the random distribution state into the aggregation state and then dissipate state in the wind field with the change of wind power. While the occurrence of precipitation could randomly remove aerosols in the precipitation area, in general, aerosol aggregation requires the meteorological support of low pressure, weak wind field and strong humidity. The dissipation of aerosol occurs with the occurrence of strong wind field and precipitation.

In the research, the process of aerosol state transfer has been deeply analysed qualitatively and quantitatively from the aspects of law, cause and interaction between urban spaces. A theoretical model of state transition process discriminance has been constructed and verified by experiments. In terms of time distribution, the overall aerosol quantity from 2015 to 2017 in this region increased, and the distribution of state transition points shows more and more winter centralized tendency, which indicates that the pollution situation in this area is increasingly more closely related to the human activities, and the meteorological factors intensify the concentrated outbreak of polluted weather. In space, we found that aerosol aggregation process was more likely to occur in urban areas than in non-urban areas, but coequally, it was also more likely to dissipate after the aggregation process than in non-urban areas. The primary cause is the frequent heat exchange of underlying surface in urban areas, which accelerates the heat exchange and air flow in turbulent processes. Meanwhile, we calculated the influence range of urban atmospheric boundary layer through the scope of urban aerosol in the state process, and proved that the urban boundary atmosphere is isolated and it affects the aerosol exchange process with non-urban areas. 